\title{
Virtual MR arthroscopy of the shoulder: image gallery with arthroscopic correlation of major pathologies in shoulder instability
}

\author{
A. Stecco - D. Volpe - N. Volpe - P. Fornara • \\ A. Castagna $\cdot$ A. Carriero
}

Received: 8 October 2007/Accepted: 19 August 2008/Published online: 7 October 2008

(C) Springer-Verlag 2008

\begin{abstract}
Background The purpose of this study was to compare virtual MR arthroscopic reconstructions with arthroscopic images in patients affected by shoulder joint instability. MR arthrography (MR-AR) of the shoulder is now a wellassessed technique, based on the injection of a contrast medium solution, which fills the articular space and finds its way between the rotator cuff (RC) and the glenohumeral ligaments. In patients with glenolabral pathology, we used an additional sequence that provided virtual arthroscopy (VA) post-processed views, which completed the MR evaluation of shoulder pathology.

Materials and methods We enrolled 36 patients, from whom MR arthrographic sequence data (SE T1w and GRE T1 FAT SAT) were obtained using a GE 0.5 T Signabefore any surgical or arthroscopic planned treatment; the protocol included a supplemental 3D, spoiled GE T1w positioned in the coronal plane. Dedicated software loaded on a work-station was used to elaborate VAs. Two radiologists evaluated, on a semiquantitative scale, the visibility of the principal anatomic structures, and then, in consensus, the pathology emerging from the VA images.
\end{abstract}

A. Stecco $(\bowtie) \cdot$ D. Volpe $\cdot$ N. Volpe · A. Carriero

Department of Radiology, Maggiore della Carità Hospital, Eastern Piedmont University, Novara, Italy

e-mail: a.stecco@libero.it

P. Fornara

Department of Orthopaedic Surgery, Maggiore della Carità Hospital, Corso Mazzini 18, Novara, Italy

A. Castagna

Department of Orthopaedic Surgery, Istituto Clinico Humanitas, Milan, Italy
Results These images were reconstructed in all patients, except one. The visualization of all anatomical structures was acceptable. VA and MR arthrographic images were fairly concordant with intraoperative findings.

Conclusions Although in our pilot study the VA findings did not change the surgical planning, the results showed concordance with the surgical or arthroscopic images.

Keywords 3D MR imaging - Magnetic resonance . MR arthrography · Virtual MR arthrography

\section{Introduction}

MR arthrography (MR-AR) is now considered the goldstandard of radiology in the evaluation of shoulder instability.

In patients with such a condition, MR-AR allows an accurate assessment of: capsulolabral complex, undersurface of the rotator cuff (RC), glenohumeral ligaments and $\mathrm{RC}$ interval [1]; moreover, the technique also gives information on eventual glenoid and humeral bone loss, for which computed tomography (CT) is the standard of reference.

The rationale of this technique is the capsular distention. This allows distinction of individual structures by improved soft-tissue contrast and physical separation obtained by the intra-articular contrast material, and allows an analysis of the distribution of contrast material "in" and "around" the joint. The combination of administration of paramagnetic contrast with a fast gradient-echo 3D T1w (SPGR 3D T1) pulse sequence provides a new method for displaying vascular and intestinal morphology [2-6]. Based on the same 
principles, virtual MR arthroscopy of the shoulder can be performed. Virtual arthroscopy (VA) provides a unique means for evaluating the complex relationship of various intra-articular structures, by virtually placing the observer within the articular space, and creating an "arthroscopiclike" illusion. The technique has the potential to enhance the diagnostic performance of MR-AR, especially for orthopedic surgeons, helping in the evaluation of crosssectional images [7].

The purpose of this investigation was to demonstrate the feasibility of virtual MR arthroscopy in comparison with the arthroscopic findings that served as the standard of reference.

\section{Materials and methods}

\section{Patients}

Between 2002 and 2006, we enrolled 36 patients (24 males, 12 females; age range 24-50 years; mean age 37 years).

All patients were scheduled for arthroscopic treatment of shoulder instability for labral, ligamentous or bone lesions. The patients had undergone MR-AR during the 3-6 months before the surgical treatment, but accepted to repeat the examination, signing an informed consent.

The study was performed according to the Declaration of Helsinki and did not require consent from the institutional ethics board, because the new MR examination had been requested by the orthopedic surgeon for the planning and had a clinical value when considering that all scans had been performed within a range of 7 days before the treatment. The arthroscopy was performed by the same surgeon in all patients. The original MR-AR findings, for which they were enlisted for surgical treatment and to be confirmed after that, are shown in Table 1 .

Table 1 List of pathologies of our patients, diagnosed before virtual arthroscopy examination and in view of the surgical arthroscopic treatment

\begin{tabular}{lr}
\hline Bankart & 21 \\
Bony Bankart & 3 \\
Perthes & 1 \\
Alpsa & 2 \\
Haghl & 3 \\
Glad & 1 \\
Hill-Sachs & 26 \\
Slap I & 3 \\
Slap II & 1 \\
Slap III & 2 \\
Slap IV & 1 \\
\hline
\end{tabular}

Imaging

MR arthrographic procedure were performed with a 22-gauge needle placed in the glenohumeral joint using percutaneous anterior palpatory-guided access. Diluted gadopentate (Magnevist) with a concentration of 0.7/100 $\mathrm{ml}$ was injected. MR imaging was performed on a GE 0.5 T Signa.

All patients were evaluated on a low-field $(0.5 \mathrm{~T})$ superconductive magnet (General Electric Signa Contour, Milwaukee, IL, USA) with three standard multiplanar MR arthrographic pulse sequences (Spin Echo T1 and GradientEcho T1 with spectral fat saturation) and a supplemental 3D spoiled gradient-echo $\mathrm{T} 1$ positioned in the oblique coronal plane (TR 22.8-28.6 ms, TE 5.7-9.9 ms, flip angle $45^{\circ}, 512 \times 256$ matrix and slice thickness $1 \mathrm{~mm}$ ).

The 3D MR data sets were post-processed by two radiologists using dedicated software (Navigator) loaded on a work-station (ADW 3.1, GE). Virtual views were constructed based on surface-rendering algorithms. Technically less demanding, they are ideal for viewing the type of "bright/dark" data provided by the contrast-enhanced 3D MR image sets. Prior to applying the surface-rendering algorithm, a threshold value was specified. Specification of the correct threshold is critical to the quality and accuracy of object depiction. The threshold was separately adapted for each individual slice. Surface models were then calculated and displayed, employing an imaginary light source. As a guideline, the threshold should correspond to the center of the signal difference between the brightest pixel found within the contrast-filled, intra-articular space and the signal of the surrounding hypointense structures. The wide field of view (FOV) of the navigator has a conic shape and simulates the geometry of optic fibers used in endoscopy. The angle of the FOV of the VA varies from $15^{\circ}$ to $60^{\circ}$ and can be adjusted by the user. With a $15^{\circ}$ angle, it will be a smaller FOV obtaining a larger image (greater zoom). A $60^{\circ}$ angle means larger FOV and smaller zoom.

\section{Analysis}

We calculated the time expended at the work-station, the number of cases successfully reconstructed and the efficacy in depicting the following six principal anatomical structures to be looked at from inside the capsule: ligaments, labrum, glenoid and humeral surfaces, capsule and RC tendon undersurface. This latter evaluation was performed, independently, by two radiologists unaware of the clinical suspicion, or of the MR-AR and surgical findings, by filling a predefined form listing five subjective levels (semiquantitative) of visualization: no visualization, poor, discrete, good and optimal. 
Table 2 Overall mean of 35 patients of the quality of visualization of the six intra-articular subsites on the virtual arthroscopy images, examined by the two blinded radiologists (RAD1 and RAD2)

\begin{tabular}{|c|c|c|c|c|c|c|c|c|c|c|}
\hline \multirow[t]{2}{*}{ Sites } & \multicolumn{2}{|c|}{ No visualization } & \multicolumn{2}{|l|}{ Poor } & \multicolumn{2}{|c|}{ Discrete } & \multicolumn{2}{|l|}{ Good } & \multicolumn{2}{|c|}{ Optimal } \\
\hline & RAD1 & RAD2 & RAD1 & RAD2 & RAD1 & RAD2 & RAD1 & RAD2 & RAD1 & RAD2 \\
\hline Ligaments & & & & & & $\mathrm{x}$ & $\mathrm{x}$ & & & \\
\hline Labrum & & & & & & & & $\mathrm{x}$ & $\mathrm{x}$ & \\
\hline Glenoid & & & & & & & & & $\mathrm{x}$ & $\mathrm{x}$ \\
\hline Humeral head & & & & & & & & & $\mathrm{x}$ & $\mathrm{x}$ \\
\hline Tendon undersurface & & & & $\mathrm{x}$ & $\mathrm{x}$ & & & & & \\
\hline Capsule & & & & & & & & & $\mathrm{x}$ & $\mathrm{x}$ \\
\hline
\end{tabular}

In a second step, the two radiologists evaluated, in consensus, the standard MR-AR versus the VA, in comparison with the arthroscopic findings (Table 3 ).

To show the results of our study, we chose an image case gallery of the lesions that we found.

\section{Results}

The time required to create virtual MR arthroscopic models ranged between 10 and $15 \mathrm{~min}$ (mean $13 \mathrm{~min}$ ).

In 35 of 36 patients, it was possible to render a virtual arthroscopic image set of the shoulder. Only in one case could VA not be performed due to a movement artefact of a non-collaborating patient. In all 35 cases, in the VA images, we were able to recognize the six anatomical districts, with a resulting mean quality of visualization of "optimal" for glenoid, humeral head and capsule sites for both radiologists; and good to optimal for the labrum, discrete to good for the ligaments, and poor to discrete for the $\mathrm{RC}$ undersurface for the first and second readers, respectively(Table 2).

The correlation among "standard" MR-AR, VA and arthroscopy (Table 2) showed that VA does not oversize the lesions, nor does it overestimate the number of lesions compared with the standard of reference. The MR arthrographic technique, however, overcalled two cases of ALPSA and bony Bankart, not corresponding to the intraoperative findings. VA showed no false positives, although it appears to be less accurate toward Bankart, HAGL and SLAP I lesions than MR-AR when compared with intraoperative findings.

The MR-AR, in our case series, had near the $100 \%$ of correspondence with the intraoperative findings, except for the already described overestimation (false positives) and for one case of missed Bankart and two of missed SLAP type I (false negatives). We show an image gallery of the correlation with the intraoperative arthroscopic findings (Figs. 1, 2; 3; 4; 5).

\section{Discussion}

$\mathrm{VA}$ is a new radiology technique that allows internal 3D anatomic structures to be to viewed by means of a complex electronic post-processing [8]. Images appear as a gray shaded surface seen from a conic point of view.

Structures demonstrated on MR-AR could be visualized on virtual MR arthroscopy in a way that is most similar to

Table 3 Types of lesion "called" by the MR and VA techniques, in comparison with the intraoperative findings

\begin{tabular}{|c|c|c|c|c|c|}
\hline Pathology & MR arthrography & Virtual arthroscopy & Arthroscopy & MR/arthroscopy & VA/arthroscopy \\
\hline Bankart & 21 & 19 & 22 & $21 / 22$ & $19 / 22$ \\
\hline Bony Bankart & 3 & 2 & 2 & $3 / 2$ & $2 / 2$ \\
\hline Perthes & 1 & 1 & 1 & $1 / 1$ & $1 / 1$ \\
\hline Alpsa & 2 & 1 & 1 & $2 / 1$ & $1 / 1$ \\
\hline Haghl & 3 & 1 & 3 & $3 / 3$ & $1 / 3$ \\
\hline Glad & 1 & 1 & 1 & $1 / 1$ & $1 / 1$ \\
\hline Hill-Sachs & 26 & 26 & 26 & $26 / 26$ & $26 / 26$ \\
\hline Slap I & 3 & 2 & 4 & $3 / 4$ & $2 / 4$ \\
\hline Slap II & 1 & 1 & 1 & $1 / 1$ & $1 / 1$ \\
\hline Slap III & 2 & 2 & 2 & $2 / 2$ & $2 / 2$ \\
\hline Slap IV & 1 & 1 & 1 & $1 / 1$ & $1 / 1$ \\
\hline
\end{tabular}


Fig. 1 Bankart lesion as seen on magnetic resonance image (MRI) (a), virtual MR

endoscopy (b) and arthroscopic images (c). Axial GRE MR shows (yellow arrow) avulsion and fragmentation of the labrum with detachment of the anteroinferior capsulolabral complex and rupture of the scapular periosteum
Fig. 2 Bony Bankart lesion as seen on magnetic resonance image (MRI) (a), virtual MR endoscopy (b) and arthroscopic images (c). Coronal MR images show avulsion fracture of the glenoid rim (yellow arrow) that carries with it the capsulolabral complex
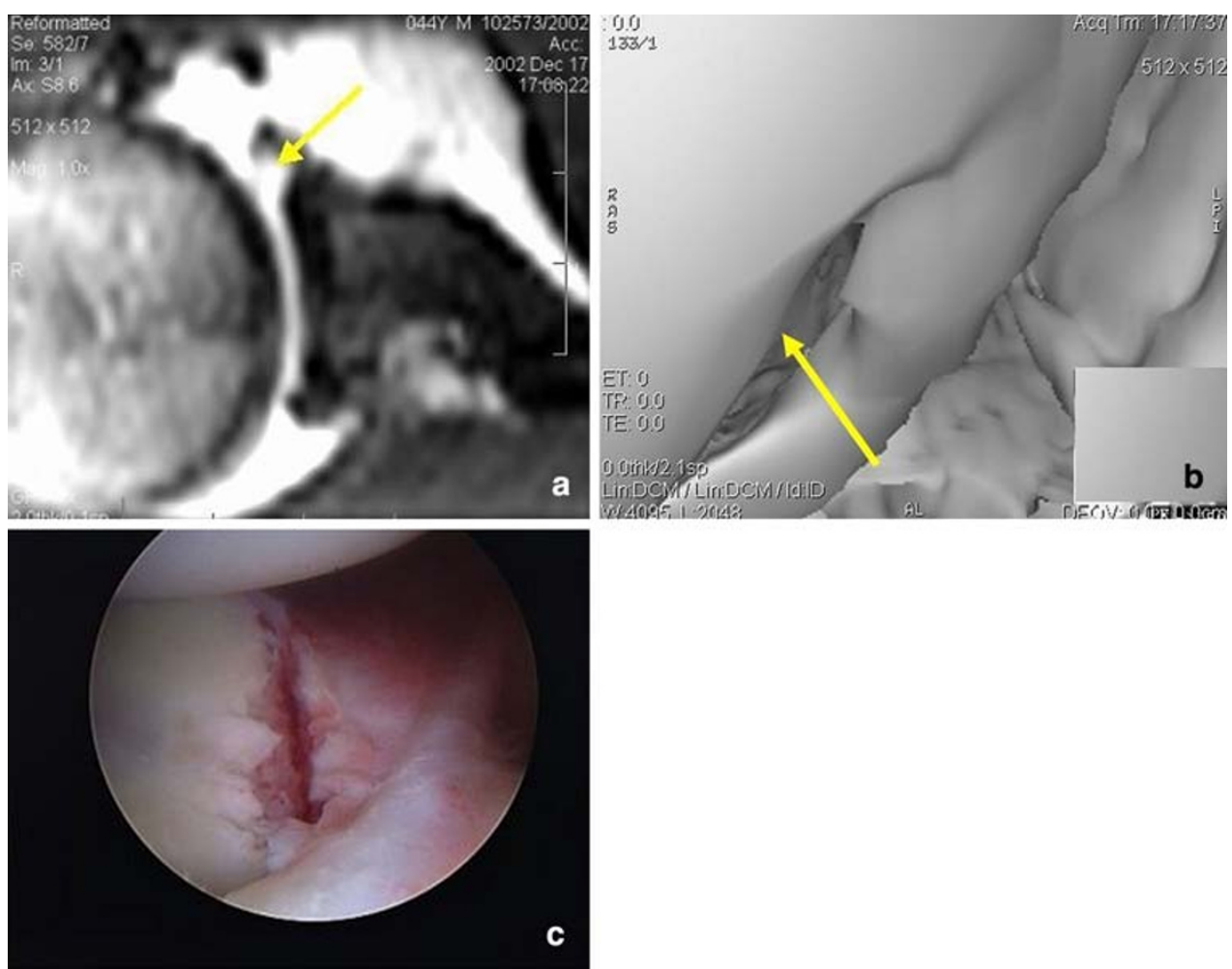

b
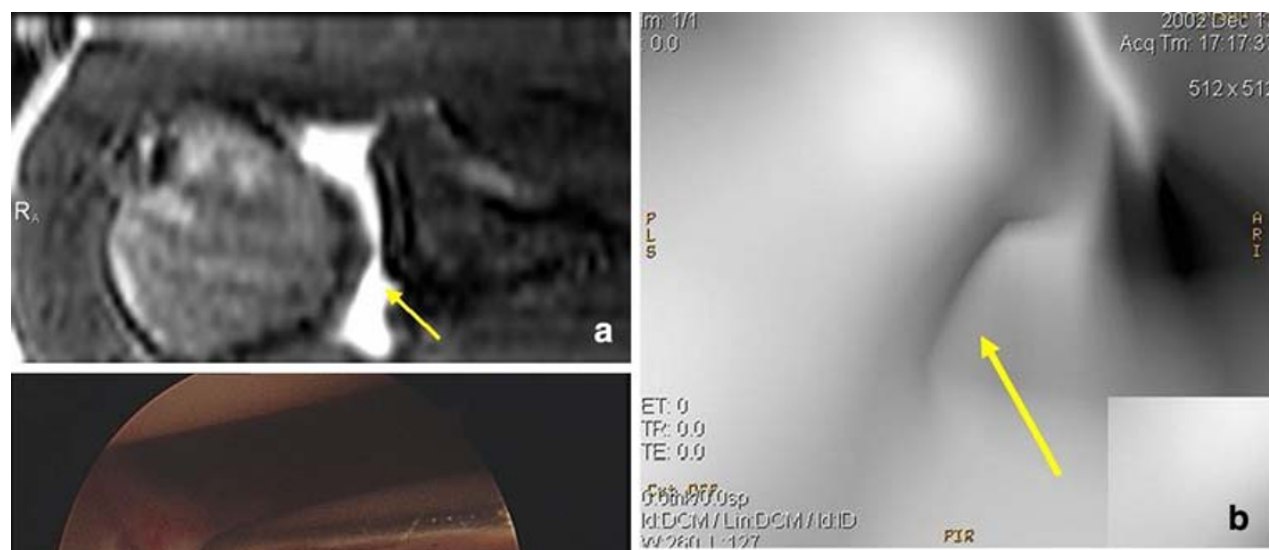

c operative arthroscopy. These include RC tears, and labral and articular cartilage abnormalities. Virtual MR shoulder arthroscopy appears to be a reliable adjunct to standard MR-AR. Anatomical structures were correctly identified [9], as seen in our case gallery.

The new technique is useful in the study of glenoid-labrum and SLAP lesions, with a near $100 \%$ reproducibility of our findings. There have been some cases of false negatives, but no false positives, for the VA images. Such false negatives can be explained by the main negative side of the technique; that is, visualization of the surface of the structures, with no possibility of assessing its internal structures. Arthroscopy is potentially affected by the same bias, but during this procedure it is possible to 
Fig. 3 HAGL lesion (humeral avulsion glenohumeral ligament) as seen on source magnetic resonance image (MRI) (a), virtual MR endoscopy (b) and arthroscopic images (c). A capsule avulsion of the capsule including the IGHL from the neck of the humerus. Axial MR image shows HAGL lesion (yellow arrow) with tearing of the axillary pouch and extension into the mid-inferior glenohumeral ligament

Fig. 4 GLAD lesion (glenolabral articular disruption) as seen on source magnetic resonance image (MRI) (a), virtual MR endoscopy (b) and arthroscopic images (c). A superficial tear of the antero-inferior labrum with an adjacent articular cartilage injury. The extent of the injury may vary from a cartilaginous flap tear to a depressed osteochondral injury of the articular cartilage and underlying bone. Axial MR image shows small corner fracture of anterior glenoid (yellow arrow) with an adjacent tear of anterior labrum
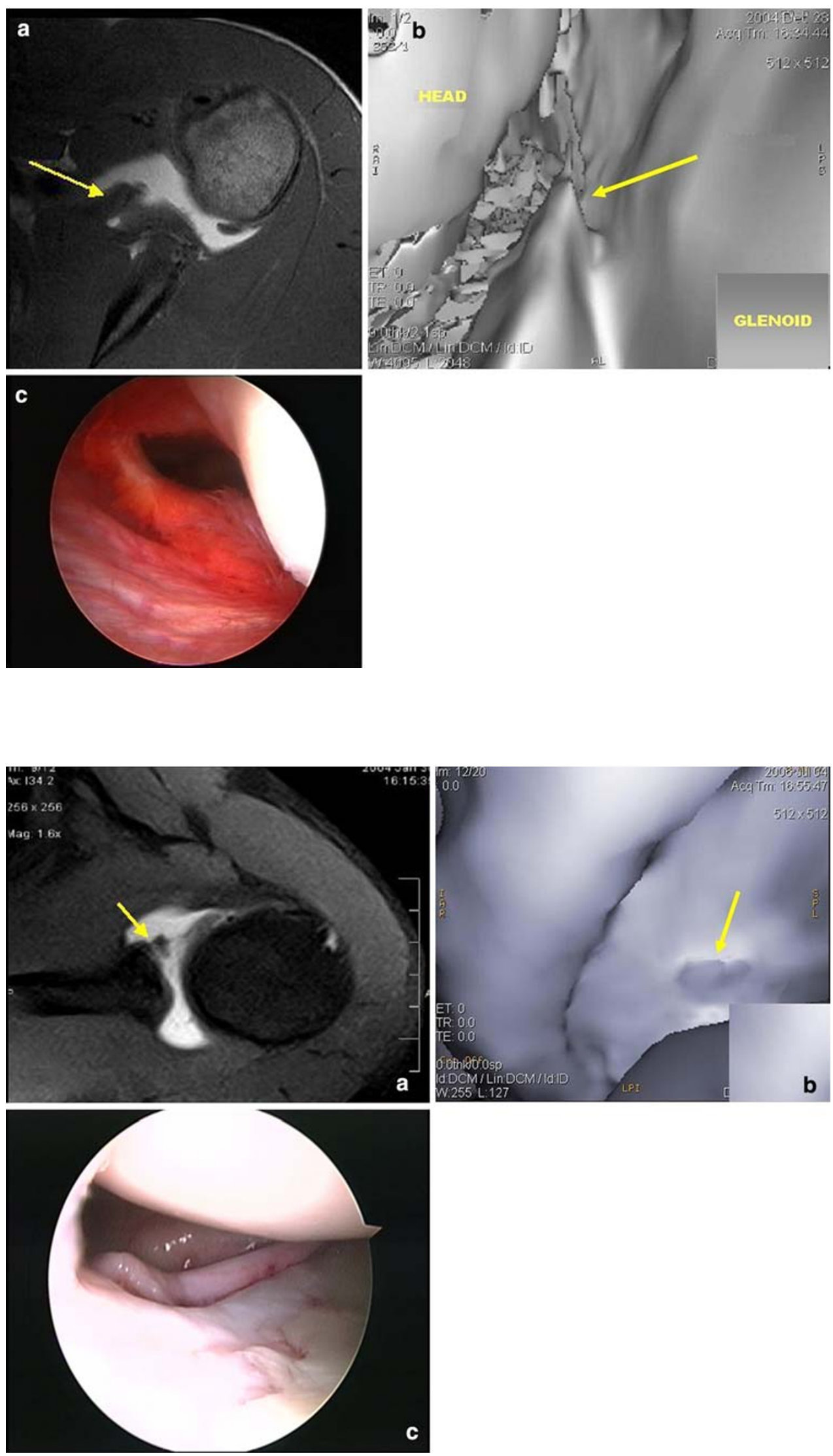
Fig. 5 SLAP lesion (superior labral, anterior and posterior tear) as seen on source magnetic resonance image (MRI), virtual MR endoscopy and arthroscopic images. Requires repetitive overhead use of the arm and varies in severity, but involves the superior portion of the glenoid labrum and, sometimes, the biceps anchor. Coronal PD MR image demonstrates irregular increased signal intensity (yellow arrow) within the superior labrum Type II SLAP lesion (a); virtual MR arthroscopy (b) and arthroscopic images (c)
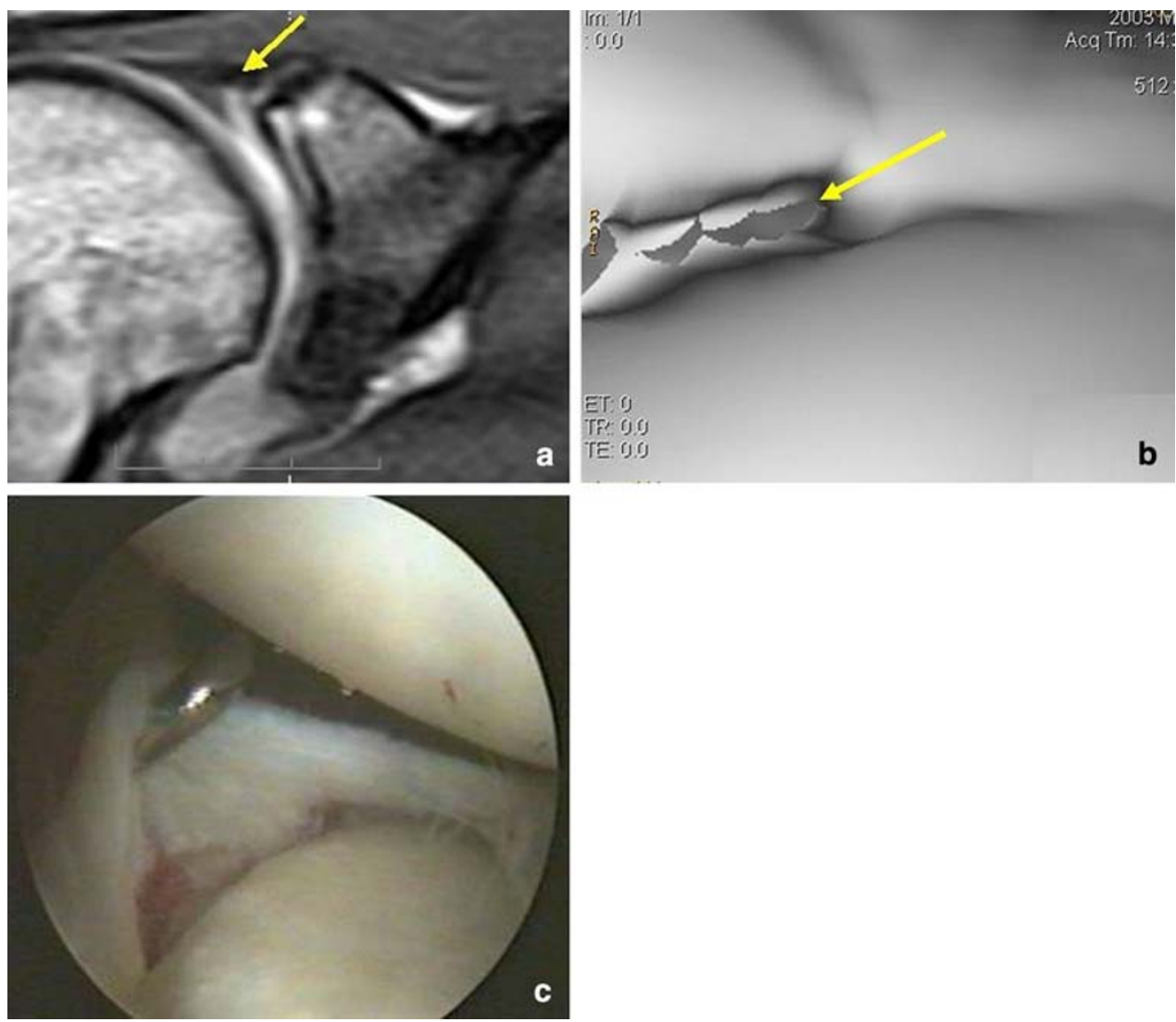

assess the viability of the structures, their aspect and thus their status.

Bony Bankart could be overcalled by MR in general, because an intraspongious signal alteration could be mistaken for a fracture; the standard of reference for bone loss evaluation is CT. VA could identify gaps in the surface or abrupt glenoid surface irregularities in the case of bony Bankart, helped by the particular viewpoint from inside. The VA images of the glenoid should be tested versus CT to assess accuracy in the qualitative and quantitative assessment of bone loss, a critical parameter that could actually switch the treatment to an open procedure.

In our pilot study, VA findings, although in some cases different with respect to the MR-AR, did not change the therapeutic planning of the surgeon, because of the experimental nature of the study. Costs did not differ much between the two, because only $8 \mathrm{~min}$ longer room occupancy was required by the VA patient (the time for the pulse sequence). However, reconstruction time spent at the work-station can be the negative side of this technique. The learning curve associated with this VA reconstruction is quite fast-it can take 2-3 days-but knowledge of shoulder anatomy is mandatory to understand the viewing position inside the articulation.

The additional diagnostic contribution of this method will have to be estimated in time; today, however, it remains true that VA can show a good view of a complex anatomy, such as that of the shoulder. This procedure may be useful in the future as a diagnostic tool and as an adjunct to clinical and surgical planning, as well as an interactive tool for learning arthroscopic anatomy and pathology.

The major bias of the present study was the low field $(0.5 \mathrm{~T})$ of the MR scanner, which resulted in less spatial resolution in the pulse sequences and bigger voxels in reconstructions (like VA algorithms); we are currently testing the protocol on a high-field (1.5 T) MR magnet.

Conflict of interest statement The authors declare that they have no conflict of interest.

\section{References}

1. Faletti C (2007) L'imaging nell'instabilità di spalla. In: Fornara $P$, Stecco A, Carriero A, Mordente G, Cisari C (eds) Spalla: clinica, imaging patologia e riabilitazione. Idelson Gnocchi, Napoli, pp 223-232

2. Applegate GR (1998) Three-dimensional MR arthrography of the shoulder: an intraarticular perspective. AJR Am J Roentgenol 171:239-241

3. Davis CP, Ladd ME, Romanowski BJ, Wildermuth S, Knoplioch JF, Debatin JF (1996) Human aorta: preliminary results with virtual endoscopy based on three-dimensional MR imaging data sets. Radiology 199:37-40 
4. Dubno B, Debatin JF, Luboldt W, Schmidt M, Hany TF, Bauerfeind P (1998) Virtual MR cholangiography. AJR 171:1547-1550

5. Leung DA, McKinnon GC, Davis CP, Pfammater T, Krestin GP, Debatin JF (1996) Breath-hold contrast-enhanced, three-dimensional MR angiography. Radiology 200:569-571

6. Luboldt W, Bauernfeind P, Steiner P, Fried M, Krestin GF, Debatin JF (1999) Preliminary assessment of three-dimensional magnetic resonance imaging for various colonic disorders. Lancet 349:1288-1291
7. Luboldt W, Debatin JF (1998) Virtual endoscopic colonography based on 3D MRI. Abdom Imaging 23:568-572

8. Neri E, Boraschi P, Caramella D et al (2000) MR virtual endoscopy of the upper urinary tract. AJR Am J Roentgenol 175:1697-1702

9. Weishaupt D, Wildermuth S, Schmid M, Hilfiker PR, Hodler J, Debatin JF (1999) Virtual MR arthroscopy: new insights into joint morphology. J Magn Reson Imaging 9:757-760 